\title{
Variable ventilation and Jensen's inequality: citation corrections
}

\author{
W. Alan C. Mutch ${ }^{1 *}$, M. Ruth Graham ${ }^{1}$ and John F. Brewster ${ }^{2}$ \\ See related research by Huhle et al., http://ccforum.biomedcentral.com/articles/10.1186/s13054-016-1216-6
}

We would like to commend Huhle and co-authors [1] for their extensive review of variable ventilation featuring citations from our pioneering work. We would suggest a couple of corrections to the cited articles, however. The authors reference important work by Venegas and colleagues [2], including Figure 4 in their manuscript. This figure explains the convex and concave portions of the sigmoidal pressure-volume curve indicating where variable ventilation has beneficial and detrimental effects, respectively, as suggested by Jensen's theorem.
No mention of Jensen's 'theorem' or 'inequality' is discussed in the paper by Venegas et al. This explanation is advanced in a paper by us and not cited ('Convexity, Jensen's inequality and benefits of noisy mechanical ventilation' [3]). The data supplement in our paper gives a comprehensive mathematical explanation for conditions where non-linear systems can and cannot benefit from the addition of noise. Mechanical ventilation is but one example where noisy life support systems may provide benefit to patients.

\section{Authors' response}

Robert Huhle, Paolo Pelosi and Marcelo Gama de Abreu

We thank Drs. Mutch, Graham, and Brewster for their interest in our review article [1], and their invaluable contribution to the field of variable ventilation. We are aware of the outstanding publication that the authors of the letter missed in our article [3].

We would like to briefly explain the reasons for not including it in our review [1].

The mechanism usually referred to as "Jensen's inequality" [3] is in fact the mathematical theorem forming the basis of stochastic resonance [4] on the convex relationship between volume and pressure of the respiratory system. Thus, while the formal more detailed description of the "Jensen's inequality" [3] contributed to understand one of the mechanisms of variable ventilation, its basis was described years before [4]. We are aware of the fact that the Venega's equation [2] does not describe the "Jensen's inequality" theorem. Rather, the equation characterizes mathematically the sigmoidal relationship between pressure and volume of the respiratory system. Thus, we combined the primary concept of stochastic

\footnotetext{
* Correspondence: wacmutch@shaw.ca

${ }^{1}$ Department of Anesthesia and Perioperative Medicine, University of

Manitoba, Winnipeg, Canada

Full list of author information is available at the end of the article
}

resonance [4] with a concave pressure-volume relationship [2] to also call attention to possible detrimental effects of variable ventilation, which were not mentioned before [3].

Most importantly, we must recognize the effort of different research groups in experimental settings showing beneficial effects of variable ventilation on respiratory function and reduction in ventilator-associated lung injury. We hope that future randomized clinical studies will determine the effects of variable ventilation on clinical outcome.

In view of these facts, and due to the journal's restrictions regarding the number of references, we included original concepts and relevant clinical and physiological studies only, including some from Dr. Mutch's group.

\footnotetext{
Authors' contributions

WACM and $\mathrm{RH}$ : conception, writing, and final approval. MRG: writing, final approval. JFB: writing and final approval. PP: writing, final approval. MGA: writing, final approval.
}

\section{Competing interests}

WACM was formerly Chief Science Officer of Biovar Life Support Inc., a company partially owned by the University of Manitoba that developed a variable ventilator. This device was not marketed. MGA was granted patents the variable pressure support ventilation, which is licensed to Dräger Medical 
AG (Lübeck, Germany). MRG and JFB declare that they have no competing interests.

\section{Author details}

'Department of Anesthesia and Perioperative Medicine, University of Manitoba, Winnipeg, Canada. ${ }^{2}$ Deparment of Statistics, University of Manitoba, Winnipeg, Canada.

\section{Published online: 17 June 2016}

\section{References}

1. Huhle R, Pelosi P, de Abreu MG. Variable ventilation from bench to bedside. Crit Care. 2016:20:62

2. Venegas JG, Harris RS, Simon BA. A comprehensive equation for the pulmonary pressure-volume curve. J Appl Physiol. 1998;84:389-95.

3. Brewster JF, Graham MR, Mutch WAC. Convexity, Jensen's inequality and benefits of noisy mechanical ventilation. J R Soc Interface. 2005;2:393-6.

4. Suki B, Alencar AM, Sujeer MK, Lutchen KR, Collins JJ, Andrade Jr JS, et al. Life-support system benefits from noise. Nature. 1998;393(6681):127-8 